\title{
Virtual Multidisciplinary Tumor Boards: A Narrative Review Focused on Lung Cancer
}

\author{
Vittorio Gebbia (D) - Aurelia Guarini - Dario Piazza - Alessandro Bertani • Massimiliano Spada • \\ Francesco Verderame · Concetta Sergi · Enrico Potenza · Ivan Fazio • Livio Blasi • \\ Alba La Sala · Gianluca Mortillaro · Elena Roz • Roberto Marchese • Maurizio Chiarenza • \\ Hector Soto-Parra · Maria Rosaria Valerio • Giuseppe Agneta • Carmela Amato • Helga Lipari • \\ Sergio Baldari · Francesco Ferraù · Alfio Di Grazia • Gianfranco Mancuso • Sergio Rizzo • \\ Alberto Firenze \\ Received: March 8, 2021 / Accepted: May 20, 2021 / Published online: June 4, 2021 \\ (C) The Author(s) 2021
}

\section{ABSTRACT}

To date, the virtual multidisciplinary tumor boards (vMTBs) are increasingly used to achieve high-quality treatment recommendations across health-care regions, which expands and develops the local MTB team to a regional or national expert network. This review describes the process of lung cancer-specific MTBs and the transition process from face-to-face tumor

\section{Gebbia $(\bowtie) \cdot$ G. Mancuso · S. Rizzo}

Medical Oncology Unit, Department of Health Promotion, Mother and Child Care, Internal Medicine and Medical Specialties, La Maddalena Clinic for Cancer, University of Palermo, Via San Lorenzo Colli n. 312D, 90100 Palermo, Italy e-mail: vittorio.gebbia@gmail.com; vittorio.gebbia@unipa.it

V. Gebbia · D. Piazza

GSTU Foundation, Palermo, Italy

A. Guarini · M. Spada

Medical Oncology Unit, Fondazione Ospedale Giglio, Cefalù, Palermo, Italy

\section{A. Bertani}

Division of Thoracic Surgery and Lung

Transplantation, Department for the Treatment and Study of Cardiothoracic Diseases and Cardiothoracic Transplantation, IRCCS Ismett, UPMC, Palermo, Italy

F. Verderame

Medical Oncology Unit, Ospedale Cervello Villa Sofia, Palermo, Italy boards to virtual ones. The review also focuses on the project organization's description, advantages, and disadvantages. Semi-structured interviews identified five major themes for MTBs: current practice, attitudes, enablers, barriers, and benefits for the MTB. MTB teams exhibited positive responses to modeled data feedback. Virtualization reduces time spent for travel, allowing easier and timely patient discussions. This process requires a secure web platform to assure the respect of patients' privacy and presents the same unanswered prob-

\section{Sergi $\cdot$ E. Potenza}

Thoracic Surgery Unit, ARNAS, Ospedale Garibaldi, Nesima, Catania, Italy

\section{Fazio}

Radiation Therapy Unit, Clinica Macchiarella, Palermo, Italy

L. Blasi

Medical Oncology Unit, Arnas Civico, Palermo, Italy

A. La Sala

Bronchial Endoscopy Unit, Arnas Civico, Palermo, Italy

G. Mortillaro

Radiation Therapy Unit, Arnas Civico, Palermo, Italy

E. Roz

Pathology Unit, La Maddalena Clinic for Cancer, Palermo, Italy

R. Marchese

Thoracic Surgery Unit, La Maddalena Clinic for Cancer, Palermo, Italy 
lems. The implementation of vMTB also permits the implementation of networks especially in areas with geographical barriers facilitating interaction between large referral cancer centers and tertiary or community hospitals as well as easier access to clinical trial opportunities. Studies aimed to improve preparations, structure, and conduct of MTBs, research methods to monitor their performance, teamwork, and outcomes are also outlined in this article. Analysis of literature shows that MTB participants discuss 5-8 cases per meeting and that the use of a vMTB for lung cancer and in particular stage III NSCLC and complex stage IV cases is widely accepted by most health professionals. Despite still-existing gaps, overall vMTB represents a unique opportunity to optimize

\section{Chiarenza}

Medical Oncology Unit, Istituto Clinico Humanitas,

Catania, Italy

H. Soto-Parra

Medical Oncology Unit, Policlinico, Catania, Italy

M. R. Valerio

Medical Oncology Unit, Policlinico, Palermo, Italy

G. Agneta

Thoracic Surgery Unit, Ospedale Cervello Villa Sofia, Palermo, Italy

\section{Amato}

Patients Advocacy "Serena a Palermo", Palermo, Italy

H. Lipari

Medical Oncology Unit, Ospedale Cannizzaro,

Catania, Italy

S. Baldari

Nuclear Medicine Unit, Department of Biomedical and Dental Sciences and Morpho-Functional

Imaging, University Hospital G. Martino, Messina, Italy

F. Ferraù

Medical Oncology Unit, Ospedale San Vincenzo, Taormina, Messina, Italy

\section{A. Di Grazia}

Radiation Oncology Unit, Istituto Clinico

Humanitas, Catania, Italy

\section{A. Firenze}

Risk Management Unit, Policlinico, University of Palermo, Palermo, Italy patient management in a patientcentered approach.

Keywords: Lung cancer; Multidisciplinary tumor boards; Virtualization; Oncology networks

\section{Key Summary Points}

\section{Why write this review?}

Multidisciplinary tumor boards are currently conducted worldwide for the optimal management of patients with cancer.

Lung cancer represents an optimal clinical setting for multidisciplinary tumor boards since it requires the participation of several health professionals. This is especially true for stage III disease.

This review discusses all clues needed to improve preparation, structure, and conduct of lung cancer multidisciplinary tumor boards, research methods to monitor their performance, teamwork, and outcomes.

\section{What can we learn from this review?}

Lung cancer multidisciplinary tumor boards allow the implementation of clinical practice guidelines use, may help capture cases for clinical trials, and improve assistance efficiency and patient care.

Virtualization can also allow the creation of a network between centers of varying relevance far from each other.

Virtualization of multidisciplinary tumor boards may represent a significant advantage since it reduces time spent traveling for both patients and health professionals, and improves participant adherence.

Virtualization may also improve patient management in settings where funding and resources may be limited. 


\section{DIGITAL FEATURES}

This article is published with digital features, including a summary slide, to facilitate understanding of the article. To view digital features for this article go to https://doi.org/10.6084/ m9.figshare.14617545.

\section{INTRODUCTION}

Although there has been significant improvement in the diagnostic and therapeutic armamentarium, lung cancer (LC) is still a major challenge worldwide with high disease-related morbidity and mortality [1]. To date, thoracic oncology care requires a complex multidisciplinary approach to assure the best quality of care [2]. Consequently, multidisciplinary tumor boards (MTBs) are an essential component of contemporary cancer care to the point that some countries established such an approach by law [3]. In 2020, Australian investigators, deeply involved in the MTB field, extensively reviewed this issue [3].

The concept of an MDT was first introduced in the UK in the 1990s, gaining more strength with the publication of the Calman-Hine plan in 1995, the radical reform of the UK's cancer services to ensure patients with cancer high and uniform standard of care, no matter where they might live $[4,5]$. A MTB includes a set of diverse specialists cooperating in the same setting, such as thoracic surgeons, medical oncologists, radiation oncologists, radiologists, pneumologists, pathologists, and molecular biologists, often defined as "core team" [4]. The extended board may also encompass nuclear medicine specialists, nutritionists, palliative and rehabilitation care physicians, patients' advocacy representatives, research nurses, or other specialists, and in academic centers also students and postdoctoral fellows [6]. Very recently, a cross-sectional qualitative interview study on breast and gynecological cancer MTB explored patients' participation in tumor conferences at six cancer centers in Germany with conflicting results [7]. Besides the fact that half of the health providers had no experience in such settings, the study concluded that routine patients' participation in MTD was not feasible due to providers' barriers and negative experiences. Only selected patients might be included in MTD looking for new opportunities and with positive experiences.

These meetings' primary purpose is to give the patient the best multidisciplinary treatment plan, shared between all the board members. The implementation of thoracic cancer-dedicated MTB aims to provide: (a) best management through diagnostic and treatment recommendations generated according to a consensus-based method and supported by guidelines, (b) appropriate and timely patients' referral, (c) identification of available clinical trials, (d) increase the awareness of cancer research, and (e) clinician education by sharing expertise from each discipline in an open environment, thereby promoting a broader knowledgebase for future care. Health governance studies on LC management suggest that integrated and multidisciplinary care reduces barriers to treatment and variation in care, improves adherence to clinical guidelines, staging, and care coordination. This strategy also significantly shortens the interval from diagnosis to treatment, and ultimately leads to a better patient experience and an increase in the quality of care for LC patients $[8,9]$. This review is based on previously conducted studies and does not contain any studies with human participants performed by any of the authors.

\section{BOARD VIRTUALIZATION AND TELEMEDICINE}

The current Covid-19 pandemic has altered the way of approaching the patient [4]. A multidisciplinary care consultation has become more challenging to achieve. Therefore, telemedicine's introduction may avoid any delay in modifying care coordination in this time of crisis [10]. Besides minimizing the rate of infection and the spread of COVID-19 disease, the concept of virtualization is catching on to the design of pathways for cancer care because they may remove geographic barriers and facilitate clinical communication and decisionmaking. Although many cancer centers and 


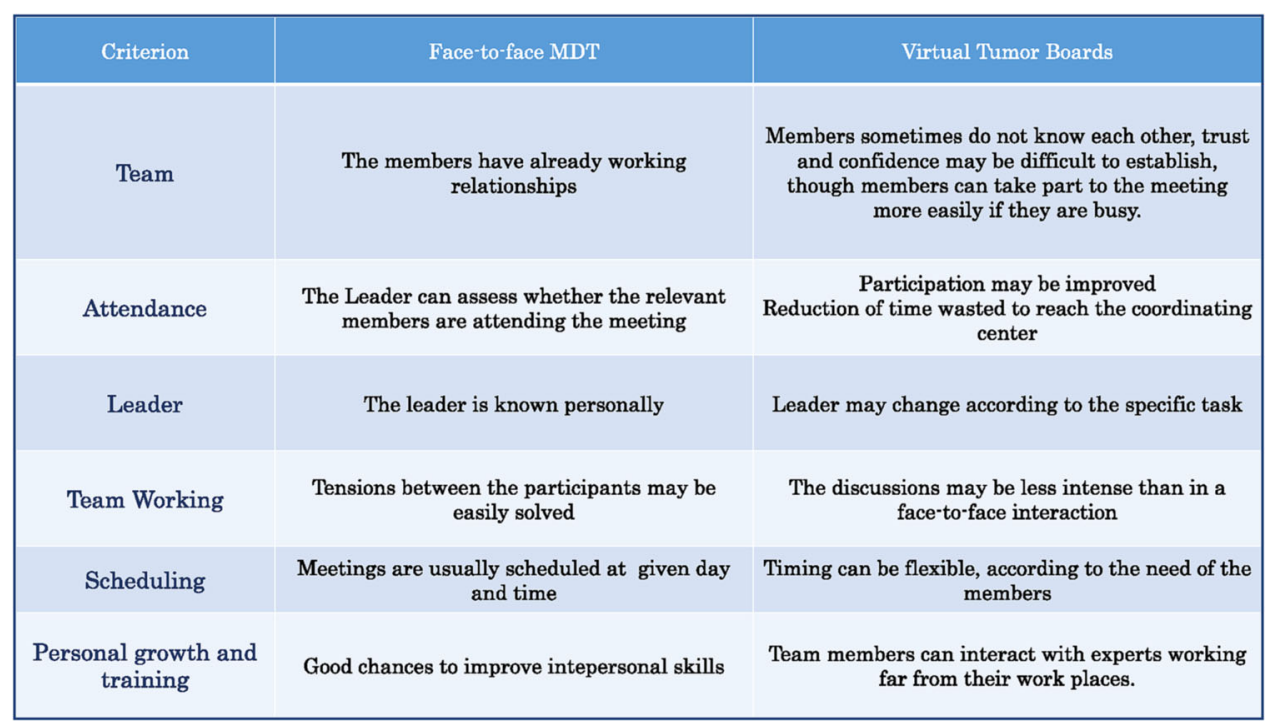

Fig. 1 Comparison between traditional face-to-face multidisciplinary tumor boards and virtual tumor boards

hospitals have local tumor boards, videoconferencing technology, boosted by the Covid-19 pandemic, has allowed the creation of virtual multidisciplinary tumor boards (vMTBs) $[11,12]$. These virtual meetings improve the collaboration between providers across geographic locations and institutions, giving proper allocation of health care resources and reducing time wasted for travel and related expenses [12, 13]. Figure 1 depicts the main characteristics of face-to-face and virtual MTBs.

The experience carried out at the University of Pittsburgh showed that meeting virtualization allows easy and efficient communication between vMTB participants at distant sites independently of the current pandemic state [12]. If adequately structured and implemented, a vMTB permits a network between high-volume academic institutions, general hospitals, and multiple satellites and community hospitals [12]. In the USA, the Duke Cancer Network created a secure web-based platform for LC-MTB connecting rural community and small hospitals with a university-based cancer center $2 \mathrm{~h}$ away as a referral center [13]. Any vMTB involved a median of ten participants that discussed no more than two cases per meeting, reporting adherence to guidelines and updated medical literature. This approach reduces time wasted for travel to reach the referral center and shortens the time to case evaluation [13]. Such a collaborative strategy strongly depends on efficient and effective communication between vMTB team members [14]. To be efficient and effective, vMTB requires a collaborative approach, appropriately leveraging the diverse expertise of LC.

\section{MOLECULAR TUMOR BOARDS}

Molecular tumor boards represent an even more recent progress in multidisciplinary care [15]. These meetings include cancer experts equipped with genomic interpretation resources to deliver accurate and timely clinical interpretations of complex genomic results for each patient within an institution or hospital network. Molecular vMTBs may provide an online forum for collaborative governance, provenance, and information sharing between experts outside a given hospital network with the potential to enhance MTB discussions. Knowledge sharing in vMTBs and communication with guidelinedeveloping organizations can lead to progress evidenced by data harmonization across resources, crowd-sourced and expert-curated genomic assertions, and more informed and explainable usage of artificial intelligence. North American researchers reviewed the 
landscape of available tools, resources, and evidence to evaluate somatic and germline tumor variants in molecular MTB [15]. In our opinion, a molecular meeting should be part of any vMTB dedicated to LC management.

\section{IMPACT ON CLINICAL OUTCOMES}

Some evidence suggests that implementing MDT discussions improves patients' outcomes, i.e., pretreatment evaluation, proper staging, adequate treatment, quality of survivorship, and overall survival [12-14]. Specchia et al. very recently reported an umbrella review on MTB's impact on all types of cancer, reporting only one systematic review on LC published several years ago in 2008 [13, 14]. However, Australian researchers recently published a scoping review of current evidence for LC-MDT data collection and analysis and its impact on clinical outcome [16-18]. The authors identified 13 studies that presented lung cancer MDT-related clinical outcomes. Three studies included MDTs from multiple tumor streams, while ten specifically focused on LC-MDTs. Overall, 11 investigations, eight of which positive, examined the effect of MDT discussion on patients' clinical outcomes. Three studies included data from MDT records and the other three from medical patients' records. Five studies used institutional registries, while the remaining six extracted data from state or national administrative datasets, with some overlap. Despite that MDT data collection is not well defined, the importance of clinical audits and data feedback and the potential for real-time analysis to improve outcomes deserve further investigation. In 2001, a single-institution study in the USA reported changes in the pretreatment evaluation, diagnosis, and treatment plans in $20-50 \%$ of cases, mainly breast cancer, presented at MTBs [19]. In 2007, the French Groupe d' Oncologie Thoracique Azuréen carried out a 1-year prospective study on 334 patients discussed during its multidisciplinary weekly meetings showing a $4.4 \%$ therapeutic discordance between the planned and the administered treatment [20]. In this series of patients, median delay of treatment was 20 days, and the overall 1-year survival rate was lower for patients with MTD discordance with no statistical significance. A study carried out in Australia on 988 LC patients registered in the cancer registry before 2011 showed how MTD discussion produced a significantly better receipt of radiotherapy among non-small-cell lung cancer (NSCLC) patients with any stage (66 vs. $33 \%, p<0.001)$ and had significantly better receipt of chemotherapy among patients with stage IV NSCLC (46 vs. $29 \%, p<0.001$ ) and palliative care (66 vs. $53 \%, p<0.001$ ) as compared to patients without MDT discussion [21]. A logistic regression analysis identified MDT discussion as an independent predictor of receiving radiotherapy, chemotherapy, and referral to palliative care but did not influence survival. In 2012, a retrospective and comparative scientific report showed that in the UK, the introduction of multidisciplinary care was associated with improved overall survival and reduced variation in survival data in various hospitals with MTB compared to the hospitals without such implementation [22]. In 2015, investigators at the MD Anderson Cancer Center and the Dana-Farber Cancer Institute published a survey on 1198 physicians to explore LC-MTB participation associations with patient survival and rate of clinical trial enrollment guideline-recommended care, and patient-reported quality [23]. This paper showed that physician engagement in MTB was associated with higher patient clinical trial participation and higher curative-intent surgery rates for stage I-II NSCLC but not with overall survival. In the same year, a national cohort study in Taiwan showed that the adjusted hazard ratio of death of stage III and IV NSCLC patients discussed at MDT was significantly lower at multivariate Cox proportional hazards model than that of patients without MDT discussion (HR = $0.87,95 \%$ confidence interval $=0.84-0.90$ ) [24]. Data were also analyzed for the propensity score as a control variable to reduce selection bias between patients with and without MDT care involvement. In 2018, an Australian cohort study included 1197 cases discussed prospectively at MTB and analyzed for adjusted survival and referral to palliative care [25]. Survival of patients discussed at MTD was higher for all stages but IIIB as compared to patients not 
discussed, but referral to palliative care was not different. Overall adjusted survival analysis for the entire cohort showed improved survival at 5 years for patients discussed at MDT (HR 0.7, range, $0.58-0.85, p<0.001)$. However, the MTD group had a lower stage IV percentage (39.3 vs. $56.1 \%$ ) and a higher proportion of early stage disease (stage I, 23.1 vs. $9.7 \%$, and stage II, 10.2 vs. $4.8 \%$, stage IIIA, 14.6 vs. $6.3 \%$ ). In 2018 , a retrospective propensity score analysis was performed on 246 consecutive Italian patients who underwent surgery for NSCLC before or after implementing an MTB [26]. Patients discussed at the MTB showed more complete staging, better TMN classification, and a longer 1-year survival rate when compared to those who were not discussed at the MTB. In 2020, a German matched-pair analysis showed a positive impact of a higher number of multidisciplinary tumor boards on the clinical outcome [27]. Patients discussed at $\geq 3$ MTD meetings had a significantly better overall survival than patients never discussed. In the same year, researchers in Taiwan carried out a retrospective study on 500 patients with stage III NSCLC to evaluate MTB's impact on survival. The median survival of patients discussed at MDT was statically longer than that of control patients $(41.2$ vs. 25.7 months; $p=0.018$ ) [28].

\section{STAGE III NSCLC: A MTB PARADIGM}

Due to the diagnostic and therapeutic algorithm's significant complexity, discussion of stage III NSCLC is the paradigm of a LC-MTB activity. Even if concurrent chemoradiotherapy plus subsequent immunotherapy are currently considered the standard of care of stage III NSCLC, however in real life, the approach is still heterogeneous across different specialists. An Italian cooperative group launched a 15-question web-based survey focusing on diagnostic/ therapeutic of stage III to 421 health professionals, including pneumologists, thoracic surgeons, and radiation and medical oncologists [29]. Some discrepancies were present in clinical locally advanced NSCLC management: the lack of a regularly planned multidisciplinary tumor board, the use of upfront surgery in multi-station stage IIIA, and territorial diffusion of chemo-radiotherapy in unresectable locally advanced NSCLC. This analysis demonstrated good compliance with international guidelines in the diagnostic workup of locally advanced NSCLC and a relationship between high clinical experience and good clinical practice. In NSCLC, precise diagnosis and adequate staging are essential to ensure uniform allocation to the best treatment strategy, but these parameters may differ among MDTs. In 2020, Dutch investigators showed a high grade of variability in $\mathrm{T}$ and $\mathrm{N}$ staging and treatment recommendation in a series of 110 patients with stage IIIA NSCLC among MDTs in different hospitals [30]. Agreement on clinical staging and treatment recommendations was rated using Randolph's free-marginal multi-rater kappa as a chance-adjusted index. Staging of T (T3 vs. T4) and N (N1 vs. N2) showed the highest variability, which reflected a wide range of additional diagnostics and influenced treatment recommendations as induction therapy and type.

\section{BARRIER AND FACILITATORS}

The implementation of MTBs, as well as the use of clinical practice guidelines, may require funding and health care resources. These limitations of such factors may negatively influence the use of MTD and are likely to be highly variable among different countries. Therefore, adequate funding and human and technical resources may allow a high-level MTD organization even in complex clinical settings.

Researchers at New South Wales, Australia, recently reported an exhaustive review on evidence-practice gaps in LC-MDC implementation [31]. Several pitfalls have been identified and grouped according to the following issues: patient, team, health service, and health system. Research and evaluation gaps comprised the lack of control condition, variation in definitions, and outcome measures. These gaps also included barriers to start pragmatic trials as a consequence of sample size and heterogeneity of MDTs. Patient-related gaps included insufficient patient-centered discussion, and a lack of 
patient evaluation of MDT meetings. Among MTB team-related pitfalls were difficulties in reaching consensus, variation in patient selection for team review, educational value, and communication within meetings. Health service and system gaps included quality outcomes and lack of cost data.

As a general rule, health professionals react positively to MDT participation and report various advantages from such strategy, but they also define areas for improvement, e.g., access to complete information and clearly identified roles for the different health professionals. A Swedish cross-sectional study explored participants' views on the meeting function, perceived benefits, and barriers among 244 health professionals [32]. Significant benefits from MTD included shared and more accurate treatment recommendations, multidisciplinary evaluation, and adherence to clinical guidelines. Significant barriers to a shared treatment recommendation were the need for supplementary investigations and inadequate pathology reports.

In 2020, investigators in the Netherlands published semi-structured interviews to identify barriers and facilitators for implementing computerized clinical decision support systems (CCDSs) in managing LC cases among 26 various health care professionals involved in MTBs [33]. Easy access to well-structured patient data, reduction of time needed to prepare cases, and MTD duration were primary facilitators for the use of CCDSs. The main barriers for adoption were incomplete or non-trustworthy output generated by the system and low capacity of MDT to adapt to local and contextual needs. Successful implementation depended on the reliability and adaptability of the CCDSs and key users' involvement in the implementation process. A simultaneous Swedish qualitative study analyzed the views on enabling and impeding factors for multidisciplinary care of health professionals participating in a nationwide vMTB on rare cancers [34]. Investigators examined the free-text opinions to identify three thematic categories: decision-making, organization, and responsibilities.

Data feedback of recommendations is another important issue. Stone et al. reported a mixed-methods study on the clinical impact of modeled data feedback at a lung cancer MTD based on pre- and post-surveys and semi-structured interviews at three Australian cancer centers [35]. Results demonstrated agreement if they reached $\geq 4$ values on a five-point Likert scale. Most participants found modeled data easy to interpret, relevant to clinical practice and the MDT, and welcomed future regular data presentations.

\section{QUALITY OF MTB}

Measuring the quality of lung cancer MDTB is a challenging goal since the complexity of LC patient management renders this outcome challenging to reach [36]. A recent publication extensively reviewed this complex issue as regard to quality metrics [36]. To date, few studies have explored this issue so far using a standardized approach. The quality of decisionmaking in MTD depends on the quality of records presented and team processes quality. Medical literature reports that MTD usually discusses 5-8 cases per meeting [35]. German investigators employed an adaptation of the observer rating scale Multidisciplinary Tumor Board Metric for the Observation of DecisionMaking (MDT-MODe) to assess the quality of the presented information and team processes in 249 cases discussed at 29 MTBs [37]. Although the quality of different aspects of information differs significantly, this study reported high mean completeness of tumor records. However, quality psychosocial details and information on patient opinion were considered low and necessitating improvement. At mixed logistic regression analysis, uncertainty and discrepancies on medical treatment discussions were linked to a higher rate for more than one treatment recommendation. Time limitation per case also represented a negative factor. A Korean study, published in 2020, retrospectively evaluated the level of agreement between IBM Watson for Oncology cognitive computing system and 405 LC-MDT recommendations concerning surgery, radiotherapy, chemo-radiotherapy, and palliative care [38]. Concordance between MDT and IBM Watson 
for Oncology occurred in $92.4 \%$ of all cases $(p<0.001)$, and concordance differed according to clinical stages. The agreement's strength was excellent (100\%) in stage IV NSCLC and extensive disease small-cell lung carcinoma (SCLC). In stage I NSCLC, the agreement rate was high $(92.4 \%)$. The concordance between MTD and artificial intelligence system was moderate in stage III NSCLC (80.8\%) and relatively lower in stage II NSCLC (83.3\%) and limited disease small-cell lung cancer (84.6\%). The discrepancy was $12.3 \%$ in surgical decisions, $16.7 \%$ of radiotherapy prescriptions, and $11.6 \%$ chemo-radiotherapy choices. No discordance occurred in metastatic disease patients. However, investigators concluded that IBM Watson for Oncology was just an assisting tool in stage I-III NSCLC and limited disease SCLC. In this stage of the disease, the patient-doctor relationship and shared decision-making may be more critical.

\section{LIABILITY}

Health professionals participating in vMTB may undergo medico-legal obligations, including patient consent, privacy, professional liability, reporting dissenting views, and duty of care [3]. Compliance with laws and regulations for data transfer and need for confidentiality agreements are mandatory. Most vMTBs employ a video platform that provides a secure web-site coupled with a secure teleconference platform to ensure patient confidentiality. Although evidence to formulate legal recommendations is scarce, authors identify the formative evidence that may guide the management of these issues in future MDTs [3].

\section{TECHNOLOGY AND MTDS}

The achievement of an accurate diagnosis and timely delivery of care demands high-quality MDT collaboration and coordination among participants. Computed clinical decision support systems (CDSSs) are significant technological progress and an integral component of today's health information technologies [39]. They assist health professionals with interpretation, diagnosis, staging, and treatment. A CDSS can be embedded throughout the patient safety continuum providing reminders, recommendations, and alerts to health care providers. Although CDSSs may reduce medical errors and improve patient outcomes, they have fallen short of their full potential. User acceptance has been identified as one of the potential reasons for this shortfall. Investigators at the University of North Carolina at Chapel Hill, USA, reported a critical analysis of health professional barriers to the adoption of computed clinical decision support systems [40]. Health professional non-acceptation of CDSS was the main barrier to technology implementation, with a possible negative effect on patients' health and well-being. The incorporation of CDSSs based on user needs/expectations in the assistance-engage model may improve the tool's use. Using CCDSs in lung cancer, MTBs may increase the efficiency of workflows supporting participants in elaborating a shared conceptual workflow of a patient case. CCDSs may help the MTB to evaluate the completeness of collected diagnostic data, stratification for the right personalized therapy according to the clinical and radiological stage and other treatment-influencing factors, and adapt care management strategies when needed. CDSSs have not been currently included in the MTB decision-making workflow, which hampers their clinical practice impact. A group of Dutch and UK researchers designed a CDSS for multidisciplinary decisionmaking in LC to support the goals mentioned earlier by presenting relevant clinical data in line with the MDT members' existing mental model structures [41]. Results achieved in simulated LC-MTBs showed that CDSSs help participants in their capacity to adjust diagnosis, staging, and classification according to the TNM system. It enabled cross-validation of diagnostic findings, surfaced discordance between diagnostic tests, and facilitated LC staging according to the diagnostic evidence, and spotting contraindications for personalized treatment selection. 


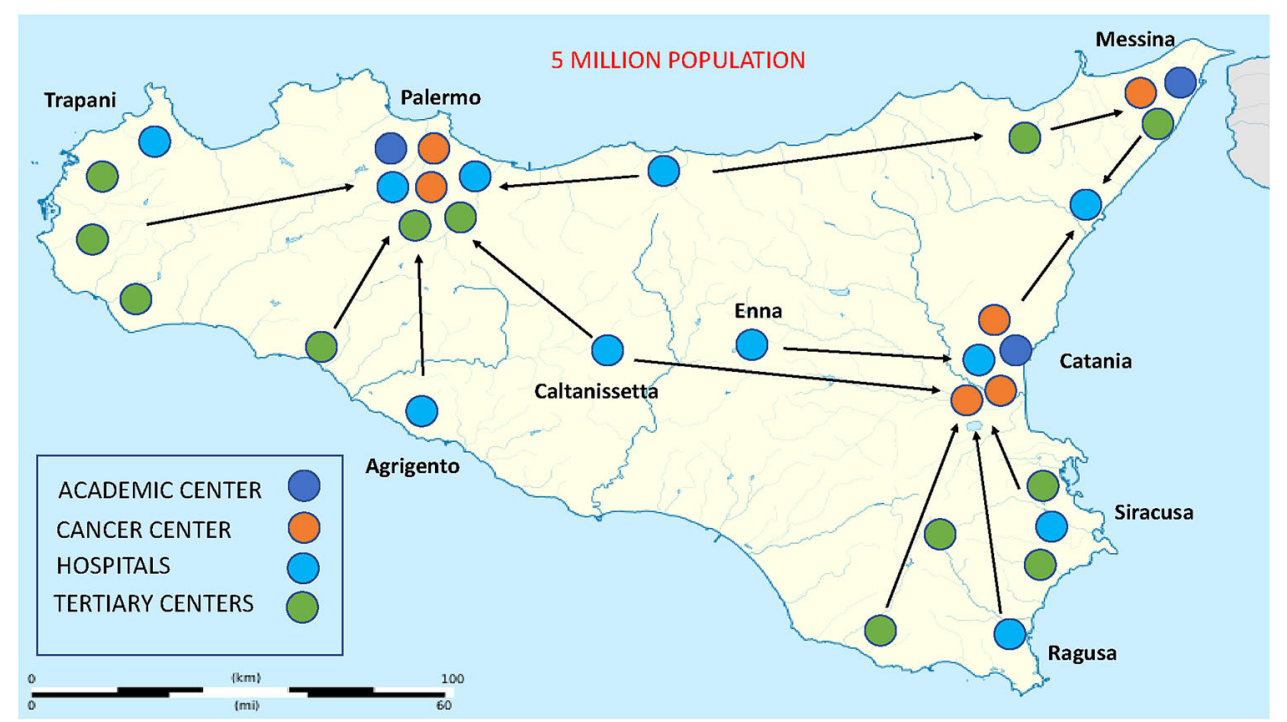

Fig. 2 Map indicating cancer centers, academic hospitals, and tertiary centers participating in the network virtual multidisciplinary tumor board. Arrows indicate usual

\section{A NETWORK VMTB EXPERIENCE}

The Covid-19 pandemic has altered the way of approaching the patient also for some limitations with clinical resources [42]. Therefore, a multidisciplinary care consultation has become more difficult. In this situation, a patient with cancer would receive a multidisciplinary patient referral dynamics and bullets represent all centers with an oncology unit according to patients volume and type of institution

consultation within several weeks. As above stated, a vMTB allowed the connection between large referral cancer centers and peripherical tertiary or community hospitals, often quite far from each other in North Carolina, USA [12].

On the other hand, an efficient vMTB organization may be essential to sustain oncological networks, especially in vast geographical areas

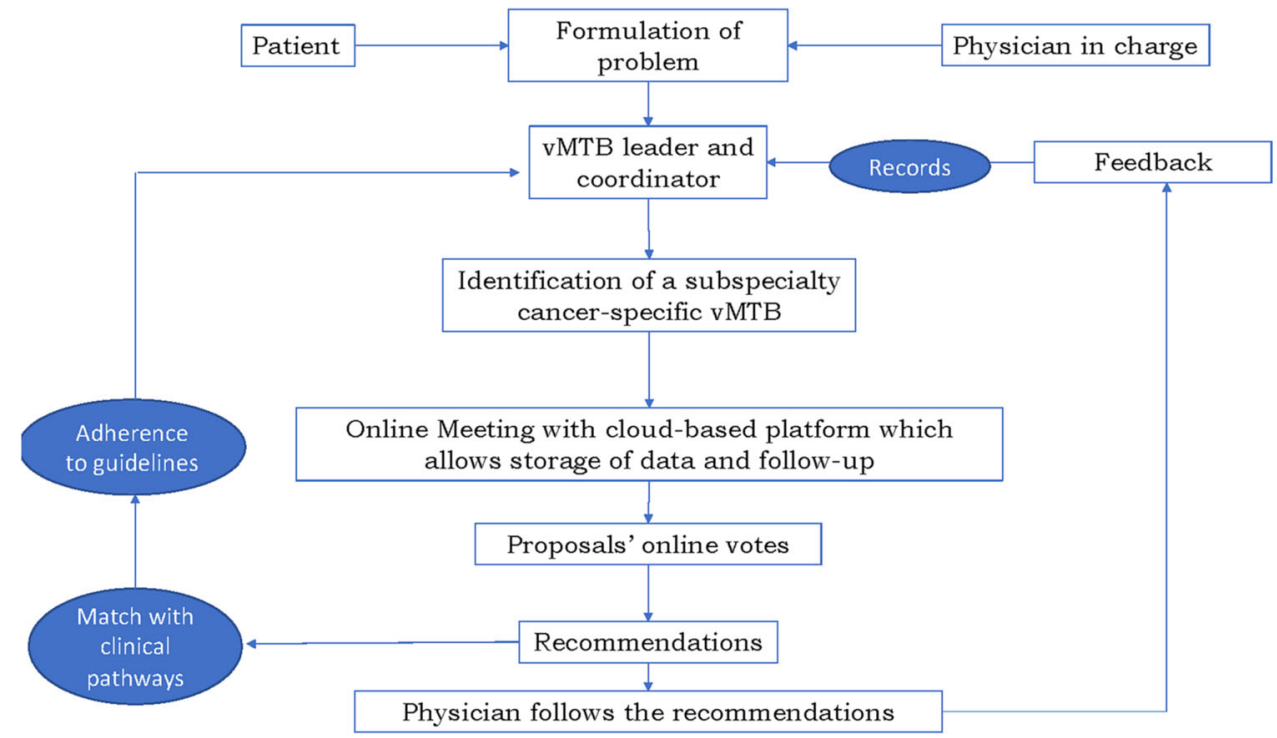

Fig. 3 Flow chart of a virtual multidisciplinary tumor board meeting 
with mobility problems. As shown in Fig. 2, many academic and cancer centers in Sicily, Italy, collaborate closely with tertiary and community hospitals, thanks to telemedicine and vMTB. The Covid-19 pandemic has improved this kind of cooperation to offer a punctual and prospective multidisciplinary clinical decision.

A clinical observation study on the efficacy and efficiency of vMTB started in July 2020 [43]. Figure 3 shows the flowchart of the vMTB process. Subspecialty cancer-specific vMTB are held at $6: 30 \mathrm{pm}$ once a week or twice a month, according to the availability of clinical cases and the participants' needs. The vMTB attendees include medical oncologists, radiation oncologists, surgeons, pathologists, molecular biologists, radiologists, nuclear medicine specialists, patients' advocacy members, and a case manager. These health professionals represent the "core team", other specialists can also participate depending on the tumor board's type and specialty. Some vMTBs may also include nutritionists, palliative care physicians, and research nurses. A useful tumor board also requires a leader to chair and run the meetings. The leader should facilitate the decision-making process by allowing the team discussion and information sharing. A coordinator will also be useful in planning the web conferences and coordinate all the members of the vMTB. Meetings occur on a web conferencing platform, provided with a specific license, with secure patient data protection and storage. Within teleconference meetings, participants employ a fully integrated platform of scalable, secure workflow solutions and apps designed to support care teams with analytics and actionable insights. This platform allows geo-localization of clinical trials, matching recommendations with updated guidelines, and access to relevant updated medical literature. The attendees invited by the guest can only attend the conference; the members can access the web platform via the URL link provided to access the meeting. Patient records are anonymized and presented by the primary oncology provider through the platform's presentation (Fig. 3). Patients have been previously informed by their doctors and have signed informed consent forms. The MTB workflow encompasses sending cases by the presenting physician to the administrator who collates and lists all the points on a platform's passwordprotected room. The clinical cases are initially presented by the patient's primary physician with relevant radiologic and pathologic findings and then discussed to obtain a final recommendation proposal. At the end of each case discussion, our web platform allows electronic and anonymous voting of the clinical decisions proposed during the meeting. If a consensus $\geq 75 \%$ of participants is achieved, each patient's chart is filled with the board's final recommendations employing the platform drop-down menu. Patients' data and decisions are then stored and may be rediscussed as follow-up progresses. The shared recommendations can be scanned and uploaded into the electronic health record system. In our experience, lung tumor board meetings usually regard the multimodality approach to stage III lung cancer, an advanced-stage disease with molecular drivers, and clinical trial opportunities. Virtual MTB organizers constituted a steering committee to validate decisions and promote scientific research (Fig. 3). After 6 months, a survey on the acceptability, appropriateness, and feasibility of vMTB was e-distributed to 74 health professionals who have been attending the meetings. We found that the majority (95\%) of respondents reported that the transition to a virtual MTD was feasible and acceptable, providing the same standard of care as face-to-face MDTs. The survey positively highlighted the impact of the project as follows: $91 \%$ on optimization of clinical information flows; $96 \%$ on equity of care; $88 \%$ on collaboration among specialists and method standardization; $77 \%$ on data security, tracking, storage, and reuse. Currently, the scientific opinions expressed have been verified as adhering to the guidelines and scientific evidence available in all the cases examined.

An important issue is the timely and accurate feedback to all MTB participants and also the general practitioners. An Australian study explored the possibility to develop and implement a standardized template for lung cancer MTB to provide clear clinical information and treatment recommendations to all participants 
and general practitioners [44]. This approach could also ensure timely information sharing between tertiary and primary care centers. The study involved 41 physicians and researchers developing a template and implementation strategy and employed a mixed-method study design using structured interviews with 61 participating general practitioners. The MDT-reporting template appears to be a feasible way of providing clinical information to general practitioners following patient presentation at a lung cancer MDT meeting. Ninety-five percent of general practitioners strongly agreed that the standardized template offered useful and relevant information, was received on time (90\%), and that the information was easy to interpret and communicate to the patient (84\%). Implementation process data show that the pre-implementation stage's investment to integrate the template into standard work practices was critical in the successful implementation.

\section{IMPLEMENTATION OF VIRTUAL MTBS}

Overall, vMTB may be implemented if some objective quality indicators are provided. The UK and Swedish researchers developed and validated a scientific instrument called "A Tumor Leadership Assessment instrument" (ATLAS) to assess and capture leadership and chairing skills in the cancer MTB setting $[45,46]$. ATLAS develops the observational assessment within 12 domains: time management, case prioritization, team involvement, discussion climate, and clarity of treatment recommendations. This validated instrument can define strengths, weaknesses, and opportunities for team development.

The time needed to prepare the clinical case represents another critical phase in the vMTB process: the case submission should occur at least $24 \mathrm{~h}$ before the meeting to allow the participants to prepare the discussion adequately. The members of the conference should be able to share their computer screen. In this manner, radiologists can share patients' scans easily. At the same time, the pathologists may be able to share reports, stains, and other information.
An essential difference between regular faceto-face sessions and vMTB is the amount of time spent in clinical case discussions. Irish analysts found that web conferences are associated with a greater time spent per case (147\%), increased participants' turn duration, and total attendance was resulting in decreased numbers of total participants per minute [47]. The most challenging clinical cases should be discussed first, when all the attendees are available, and each case should be presented within a given time. The data recording should be goal-oriented: all the participants should have access to patients' information and may be able to edit them. Some web applications like Research Electronic Data Capture (REDCap) are used to document the vMTB meetings, and register patient information such as diagnosis, histopathology, and the board's decisions [48].

\section{CONCLUSIONS}

Virtual MTB can extend and facilitate the advantages of traditional MTB. Besides the fact that in the COVID-19 pandemic era they may represent useful tools to reduce interpersonal contact and virus spread, virtualization allows health providers to participate and communicate in a much more manageable way. Since no time is spent for travel, the involvement of all figures and participants is strongly enhanced. However, virtual meetings may not necessarily substitute for the traditional face-to-face tumor boards because there are still some advantages when the participating members get to know each other personally. The interaction between diverse specialists with information and decision sharing is fundamental for an optimal clinical recommendation.

Virtual MTB may overcome the traditional tumor boards' hurdles: (a) the participating members' engagement to the web conferences is enhanced; (b) geographical barriers are eliminated; (c) patients receive a multidisciplinary care treatment plan without delay; (d) data can be analyzed automatically and stored in the cloud-based platform; (e) clinical trial referral is improved, and (f) scientific research encouraged. Although telemedicine has facilitated 
patient management, more studies are needed to validate this new clinical practice. MDT data collection and linkage are not standardized and not routine with few exceptions, although data collection and feedback are recommended explicitly by at least one statutory body. In conclusion, vMTB permits optimal per-patient decision-making as treatment options become ever-more specialized in the era of biomarkerdriven therapeutic strategies.

\section{ACKNOWLEDGEMENTS}

The authors are deeply grateful to Aurelia Guarini MD, who dedicated much effort in managing the lung cancer virtual tumor board and this review as part of her post-doctoral fellowship.

Funding. No funding or sponsorship was received for this study or publication of this article.

Authorship. All named authors meet the International Committee of Medical Journal Editors (ICMJE) criteria for authorship for this article, take responsibility for the work's integrity as a whole, and have given their approval for this version to be published.

Authorship contribution. VG, AG, DP: writing the paper, VG and AF: conceptualization, CA: assuring patient advocacy opinion. All other authors participated in the boards and to implementation and organizational efforts and reviewed and approved the manuscript.

Disclosures. Vittorio Gebbia, Aurelia Guarini, Dario Piazza, Alessandro Bertani, Massimiliano Spada, Francesco Verderame, Concetta Sergi, Enrico Potenza, Ivan Fazio, Livio Blasi, Alba La Sala, Gianluca Mortillaro, Elena Roz, Roberto Marchese, Maurizio Chiarenza, Hector Soto-Parra, Maria Rosaria Valerio, Giuseppe Agneta, Carmela Amato, Helga Lipari, Sergio Baldari, Francesco Ferraù, Alfio Di Grazia, Gianfranco Mancuso, Sergio Rizzo and Alberto Firenze have nothing to disclose.
Compliance with Ethics Guidelines. This article is based on previously conducted studies and does not contain any studies with human participants performed by any authors.

Open Access. This article is licensed under a Creative Commons Attribution-NonCommercial 4.0 International License, which permits any non-commercial use, sharing, adaptation, distribution and reproduction in any medium or format, as long as you give appropriate credit to the original author(s) and the source, provide a link to the Creative Commons licence, and indicate if changes were made. The images or other third party material in this article are included in the article's Creative Commons licence, unless indicated otherwise in a credit line to the material. If material is not included in the article's Creative Commons licence and your intended use is not permitted by statutory regulation or exceeds the permitted use, you will need to obtain permission directly from the copyright holder. To view a copy of this licence, visit http://creativecommons.org/licenses/by$\mathrm{nc} / 4.0 /$.

\section{REFERENCES}

1. Barta JA, Powell CA, Wisnivesky JP. Global epidemiology of lung cancer. Ann Glob Health. 2019; 22;85(1):8.

2. Spira A, Ettinger DS. Multidisciplinary management of lung cancer. N Engl J Med. 2004;350(4):379-92.

3. Karas PL, Rankin NM, Stone E. Medicolegal Considerations in Multidisciplinary Cancer Care. JTO Clin Res Rep. 2020;1(4):100073.

4. Calman-Hine Report. A Report by the Expert Advisory Group on Cancer to the Chief Medical Officers of England and Wales. A Policy Framework for Commissioning Cancer Services - The Calman-Hine Report. London: Department of Health; 1995.

5. Morris E, Haward RA, Gilthorpe MS, Craigs C, Forman D. The impact of the Calman-Hine report on the processes and outcomes of care for Yorkshire's colorectal cancer patients. Br J Cancer. 2006;95(8): 979-85. 
6. Lesslie M, Parikh JR. Implementing a multidisciplinary tumor board in the community practice setting. Diagnostics (Basel). 2017;7(4):55-8.

7. Heuser C, Diekmann A, Schellenberger B, Bohmeier B, Kuhn W, Karbach U, et al. Patient Participation in Multidisciplinary Tumor Conferences from the Providers' Perspective: Is It Feasible in Routine Cancer Care? J Multidiscip Healthc. 2020;13: 1729-39.

8. Kowalczyk A, Jassem J. Multidisciplinary team care in advanced lung cancer. Transl Lung Cancer Res. 2020;9(4):1690-8.

9. Denton E, Conron M. Improving outcomes in lung cancer: the value of the multidisciplinary health care team. J Multidiscip Healthc. 2016;9:137-44.

10. Sirintrapun SJ, Lopez AM. Telemedicine in Cancer Care. Am Soc Clin Oncol Educ Book. 2018;38: 540-5.

11. Shea CM, Teal R, Haynes-Maslow L, McIntyre M, Weiner BJ, Wheeler SB, et al. Assessing the feasibility of a virtual tumor board program: a case study. J Healthc Manag. 2014;59(3):177-93.

12. Dharmarajan $H$, Anderson JL, Kim S, Sridharan S, Duvvuri U, Ferris RL, et al. Transition to a virtual multidisciplinary tumor board during the COVID19 pandemic: University of Pittsburgh experience. Head Neck. 2020;42(6):1310-6.

13. Stevenson MM, Irwin $\mathrm{T}$, Lowry $\mathrm{T}$, Ahmed $\mathrm{MZ}$, Walden TL, Watson M, Sutton L. Development of a virtual multidisciplinary lung cancer tumor board in a community setting. J Oncol Pract. 2013;9(3): e77-80.

14. Popat S, Navani N, Kerr KM, Smit EF, Batchelor TJP, Van Schil P, Senan S, McDonald F. Navigating diagnostic and treatment decisions in non-small cell lung cancer: expert commentary on the multidisciplinary team approach. Oncologist. 2021;26(2):e306-15.

15. Rao S, Pitel B, Wagner AH, Boca SM, McCoy M, King I, et al. Collaborative, Multidisciplinary Evaluation of Cancer Variants Through Virtual Molecular Tumor Boards Informs Local Clinical Practices. JCO Clin Cancer Inform. 2020;4:602-13.

16. Specchia ML, Frisicale EM, Carini E, Di Pilla A, Cappa D, Barbara A, et al. The impact of tumor board on cancer care: evidence from an umbrella review. BMC Health Serv Res. 2020;20(1):73-82.

17. Coory M, Gkolia P, Yang IA, Bowman RV, Fong KM. Systematic review of multidisciplinary teams in the management of lung cancer. Lung Cancer. 2008;60(1):14-21.
18. Stone E, Rankin N, Currow D, Fong KM, Phillips JL, Shaw T. Optimizing lung cancer MDT data for maximum clinical impact-a scoping literature review. Transl Lung Cancer Res. 2020;9(4):1629-38.

19. Chang JH, Vines E, Bertsch H, Fraker DL, Czerniecki $\mathrm{BJ}$, Rosato EF, et al. The impact of a multidisciplinary breast cancer center on recommendations for patient management: the University of Pennsylvania experience. Cancer. 2001;91(7):1231-7.

20. Leo F, Venissac N, Poudenx M, Otto J, Mouroux J, Groupe d'Oncologie Thoracique Azuréen. Multidisciplinary management of lung cancer: how to test its efficacy? J Thorac Oncol. 2007;2(1):69-72.

21. Boxer MM, Vinod SK, Shafiq J, Duggan KJ. Do multidisciplinary team meetings make a difference in the management of lung cancer? Cancer. 2011;117(22):5112-20.

22. Kesson EM, Allardice GM, George WD, Burns HJ, Morrison DS. Effects of multidisciplinary team working on breast cancer survival: retrospective, comparative, interventional cohort study of 13722 women. Brit Med J. 2012;344:e27118.

23. Kehl KL, Landrum MB, Kahn KL, Gray SW, Chen $\mathrm{AB}$, Keating NL. Tumor board participation among physicians caring for patients with lung or colorectal cancer. J Oncol Pract. 2015;11(3):e267-278.

24. Pan CC, Kung PT, Wang YH, Chang YC, Wang ST, Tsai WC. Effects of multidisciplinary team care on the survival of patients with different stages of nonsmall cell lung cancer: a national cohort study. PLOS ONE. 2015;10(5):e0126547.

25. Stone E, Rankin N, Kerr S, Fong K, Currow DC, Phillips J, et al. Does presentation at multidisciplinary team meetings improve lung cancer survival? Findings from a consecutive cohort study. Lung Cancer. 2018;124:199-204.

26. Tamburini N, Maniscalco P, Mazzara S, Maietti E, Santini A, Calia N, et al. Multidisciplinary management improves survival at 1 year after surgical treatment for non-small-cell lung cancer: a propensity score-matched study. Eur J Cardiothorac Surg. 2018;53(6):1199-204.

27. Freytag M, Herrlinger U, Hauser S, Bauernfeind FG, Gonzalez-Carmona MA, Landsberg J, et al. Higher number of multidisciplinary tumor board meetings per case leads to improved clinical outcome. BMC Cancer. 2020;20(1):355.

28. Hung HY, Tseng YH, Chao HS, Chiu CH, Hsu WH, Hsu HS, et al. Multidisciplinary team discussion results in survival benefit for patients with stage III non-small-cell lung cancer. PLoS ONE. 2020;15(10): e0236503. 
29. Bruni A, Giaj-Levra N, Ciammella P, Maragna V, Ferrari K, Bonti V, et al. Management of locally advanced non-small cell lung cancer in the modern era: a national Italian survey on diagnosis, treatment and multidisciplinary approach. PLoS ONE. 2019;14(11):e0224027.

30. Hoeijmakers F, Heineman DJ, Daniels JM, Beck N, Tollenaar RAEM, Wouters MWJM, MDT Study Group, et al. Variation between multidisciplinary tumor boards in clinical staging and treatment recommendations for patients with locally advanced non-small cell lung cancer. Chest. 2020;158(6):2675-87.

31. Rankin NM, Fradgley EA, Barnes DJ. Implementation of lung cancer multidisciplinary teams: a review of evidence-practice gaps. Transl Lung Cancer Res. 2020;9(4):1667-79.

32. Rosell L, Alexandersson N, Hagberg O, Nilbert M. Benefits, barriers and opinions on multidisciplinary team meetings: a survey in Swedish cancer care. BMC Health Serv Res. 2018;18(1):249.

33. Klarenbeek SE, Schuurbiers-Siebers OCJ, van den Heuvel MM, Prokop M, Tummers M. Barriers and facilitators for implementation of a computerized clinical decision support system in lung cancer multidisciplinary team meetings-a qualitative assessment. Biology (Basel). 2020;10(1):9.

34. Rosell L, Wihl J, Nilbert M, Malmström M. Health professionals' views on key enabling factors and barriers of national multidisciplinary team meetings in cancer care: a qualitative study. J Multidiscip Healthc. 2020;13:179-86.

35. Stone E, Rankin NM, Vinod SK, Nagarajah M, Donnelly C, Currow DC, Fong KM, Phillips JL, Shaw T. Clinical impact of data feedback at lung cancer multidisciplinary team meetings: a mixed methods study. Asia Pac J Clin Oncol. 2020;16(1):45-55.

36. Wakeham E, Odell D, Samson P. Measuring the quality of multidisciplinary thoracic oncology care. J Thorac Dis. 2019;11(Suppl 4):S562-5.

37. Hahlweg P, Didi S, Kriston L, Härter M, Nestoriuc Y, Scholl I. Process quality of decision-making in multidisciplinary cancer team meetings: a structured observational study. BMC Cancer. 2017;17(1): 772 .

38. Kim MS, Park HY, Kho BG, Park CK, Oh IJ, Kim YC, et al. Artificial intelligence and lung cancer treatment decision: agreement with recommendation of multidisciplinary tumor board. Transl Lung Cancer Res. 2020;9(3):507-14.
39. Sutton RT, Pincock D, Baumgart DC, Sadowski DC, Fedorak RN, Kroeker KI. An overview of clinical decision support systems: benefits, risks, and strategies for success. NPJ Digit Med. 2020;3:17.

40. Khairat S, Marc D, Crosby W, Al SA. Reasons for physicians not adopting clinical decision support systems: critical analysis. JMIR Med Inf. 2018;6(2): e24.

41. Pluyter JR, Jacobs I, Langereis S, Cobben D, Williams S, Curfs J, van den Borne B. Looking through the eyes of the multidisciplinary team: the design and clinical evaluation of a decision support system for lung cancer care. Transl Lung Cancer Res. 2020;9(4):1422-32.

42. Smotherman C, Norez D, Austin-Datta R, Yaghjyan L. COVID-19 in patients with cancer. Lancet Oncol. 2020;21(12):e539.

43. Blasi L, Bordonaro R, Serretta V, Piazza D, Firenze A, Gebbia V. Virtual Clinical and Precision Medicine Tumor Boards: a Cloud-based Platform-mediated Implementation of Multi-disciplinary Reviews among Oncology Centers in the Covid-19 Era. JMIR Res Protoc. 2021. (in press)

44. Rankin NM, Collett GK, Brown CM, Shaw TJ, White $\mathrm{KM}$, Beale PJ, et al. Implementation of a lung cancer multidisciplinary team standardised template for reporting to general practitioners: a mixed-method study. Brit Med J Open. 2017;7(12):e018629.

45. Jalil R, Soukup T, Akhter W, Sevdalis N, Green JSA. Quality of leadership in multidisciplinary cancer tumor boards: development and evaluation of a leadership assessment instrument (ATLAS). World J Urol. 2018;36(7):1031-8.

46. Wihl J, Rosell L, Bendahl PO, De Mattos CBR, Kinhult $S$, Lindell $G$, et al. Leadership perspectives in multidisciplinary team meetings; observational assessment based on the ATLAS instrument in cancer care. Cancer Treat Res Commun. 2020;25: 100231.

47. Kane B, Luz S, O'Briain DS, McDermott R. Multidisciplinary team meetings and their impact on workflow in radiology and pathology departments. BMC Med. 2007;5:15.

48. Rangabashyam M, Wee HE, Wang W, Mueller S, Karim KABA, Skanthakumar $T$, et al. Electronic tumor board presentations as the basis for the development of a head and neck cancer database. Laryngosc Investig Otolaryngol. 2020;5(1):46-54. 